\title{
Identification of optimum segment in single channel EEG biometric system
}

\author{
Muhammad Afif Hendrawan, Pramana Yoga Saputra, Cahya Rahmad \\ Department of Information Technology, Politeknik Negeri Malang, Indonesia
}

\begin{tabular}{l} 
Article Info \\
\hline Article history: \\
Received May 21, 2021 \\
Revised Jul 30 2021 \\
Accepted Aug 6, 2021
\end{tabular}

\section{Keywords:}

Biometric

EEG

LDA

SVM

\begin{abstract}
Nowadays, biometric modalities have gained popularity in security systems. Nevertheless, the conventional commercial-grade biometric system addresses some issues. The biggest problem is that they can be imposed by artificial biometrics. The electroencephalogram (EEG) is a possible solution. It is nearly impossible to replicate because it is dependent on human mental activity. Several studies have already demonstrated a high level of accuracy. However, it requires a large number of sensors and time to collect the signal. This study proposed a biometric system using single-channel EEG recorded during resting eyes open (EO) conditions. A total of 45 EEG signals from 9 subjects were collected. The EEG signal was segmented into 5 second lengths. The alpha band was used in this study. Discrete wavelet transform (DWT) with Daubechies type 4 (db4) was employed to extract the alpha band. Power spectral density (PSD) was extracted from each segment as the main feature. Linear discriminant analysis (LDA) and support vector machine (SVM) were used to classify the EEG signal. The proposed method achieved $86 \%$ accuracy using LDA only from the third segment. Therefore, this study showed that it is possible to utilize single-channel EEG during a resting EO state in a biometric system.
\end{abstract}

This is an open access article under the CC BY-SA license.

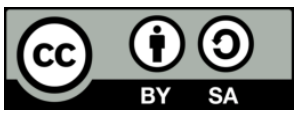

\section{Corresponding Author:}

Muhammad Afif Hendrawan

Department of Information Technology

Politeknik Negeri Malang

Soekarno-Hatta St. No. 9 Malang City, East Java, Indonesia

Email: afif.hendrawan@gmail.com

\section{INTRODUCTION}

One of the critical factors in asset protection is a security system. The security system should meet several requirements, such as difficult to penetrate, implementing the authentication method, and authorization to provide a secure security system. The authentication method is an essential part of the security system. It verifies the user before being able to access the assets. The combination of username and password is the most widely adopted authentication method. However, it has been proved that the username and password had many vulnerabilities. Their combination is easy to forget, and it can be stolen using a dictionary brute force attack. Another approach is the concept of "something you have" [1]. It means the proof of identity is represented by an object which the user has. It can be an identity card, smart card, radio frequency identification device (RFID), and so on. There are some issues according to the "something you have" concept. The object can be stolen, damaged, and it is not flexible.

Due to advances in technology, it is possible to collect human traits as a biometric modality [2], [3]. Researchers tried to study the possibilities of fingerprint, voice, face, iris, and palm print in biometric authentication systems [2]-[6]. Fingerprint modality is the most common commercial grade biometric system 
in the market. It is easy to collect the data and requires low computational cost in practice. Nonetheless, fingerprints face some issues in security. One of which is it is possible to replicate a real finger using an artificial finger [7].

Moreover, another market-ready biometric authentication system is also facing the same issue. Voice modality can be imitated using playback spoofing [5]. Face, iris, and palm print image-based traits can be replicated using a high-resolution image [8]. The main reason these modalities can be imitated is because they are exposed. Therefore, to provide a high-security biometric authentication system, invisible modalities that are not exposed from outside are needed.

Human biosignal is a potential modality to resolve that issue. It is currently gaining popularity as a modality for nonmedical use [9]-[12]. Biosignal is an electrical signal produced by human body activity. Electroencephalogram (EEG) is an example of a biosignal. It captures the electrical signal from brain activity. Marcel and Millan [13] found that the EEG signal is unique among the others. Therefore, it can be used as a biometric authentication modality. EEG has several advantages compared to conventional modality. First, EEG is invisible, and it can not be captured using remote sensing [14]. Second, the EEG signal is dependent on the mental condition of an individual [15]. A mental condition such as stress, mood, and pressure will affect the EEG signal. Third, the EEG signal proved that the signal is coming from a living individual [1].

Several studies have been proposed to use EEG as a biometric authentication modality. It can be divided into three main categories based on their stimuli. The first category is based on resting state, the second category is visual stimuli, and the last is based on motor stimuli. Paranjape et al. [16] is a notable study in the early stage of this study. They use eight-channel EEG at F7, F8, T3, T4, T5, T6, P3, and P4 positions according to the 10-20 system. Autoregressive (AR) model features and discriminant analysis were employed to classify 40 subjects based on resting-state in eye-opening and eye-closed conditions. The study achieved $80 \%$ accuracy by using a $50 \%$ data sample as training data and $100 \%$ accuracy by using all data as training data. However, [16] employs a high number of epoch and high order of AR to achieve that accuracy. The effect of the resting-state also wasn't clearly reported. Palaniappan and Mandic [17] proposed a visual stimuli method during the EEG recording session. Subjects are required to remember a picture that shows in the visual stimuli. 61 EEG channel was utilized in [17], and it gains $98.12 \%$ accuracy by using extended nearest neighbor (ENN). The multiple signal classification (MUSIC) algorithm was employed in [17] to extract EEG features, but only the power spectrum component was used.

The EEG motor imaginary (EEGMMIDB) dataset from PhysioNet [18] has become popular in biometric studies. The data was initially designed for a brain-computer interface (BCI) study. EEGMMIDB consists of 4 tasks of motor imaginary, and two baseline conditions. Resting-state with eye open (EO) and eye close (EC) are the baseline conditions in EEGMMIDB [18]. Only resting EO and resting EC widely reported were used in the biometric study [19]-[22]. Rocca et al. [19] employed EO and EC baseline from the EEGMMIDB dataset. They proposed spectral coherence connectivity, a multi-brain region fuse spectral coherence. Their study showed that connectivity between brain regions improves EEG-based biometric performance rather than power spectrum estimation from a single brain region. Moreover, Fraschini et al. [20] continued studying brain connectivity based on [19] by proposing eigenvector centrality instead of whole functional brain connectivity. They applied Euclidean distance to compare each feature set, and they used false acceptance rate (FAR) and false rejection rate (FRR) as an evaluator. Fraschini et al. [20] reported that the equal error rate (EER) from the gamma band is 0.044, and EER from high beta is 0.102. It was also reported that a low beta band showed a lower rate with 0.144 of EER. Thomas and Vinod [21] proposed non-interconnectivity brain function in their study from the same dataset. They employ sample entropy (SampEn) from 5 band signals, delta, theta, alpha, beta, and gamma. Their study reported an average correct recognition rate of $98.31 \%$. They also reported the extracted power spectral density (PSD) from SampEn enhanced correction rate up to $99.7 \%$. Kang et al. [22] tried to reduce the number of channels. The frontal channel contains eye movement activity that generates an electrooculogram (EOG) signal during EO state [15], [22], [23]. Moreover, the occipital channel generated a burst of high alpha signals that inconsistently appear during EC state [22]. Therefore, they excluded 22 channels from frontal and occipital regions due to noise issues. As a final result, Kang et al. [22] only employ 34 channels in their study. Ten single-channel and ten multi-channel features, including PSD, were extracted. They reported $0.73 \%$ of EER and $1.80 \%$ of EER during EO and EC, respectively.

The previous studies showed outstanding results by using multiple channels. However, a fewer number of channels or even single-channel EEG is more reasonable in practice. The study of single-channel EEG is rarely reported. Suppiah and Vinod [15] collected the EEG signal from the O2 channel during the EC state. They reported that the alpha channel is an effective band combined with PSD features to distinguish oneself from others. Fisher's linear discriminant analysis (FLDA) was employed to examine the proposed method. However, Suppiah and Vinod [15], reported that the proposed method requires more than 5 seconds 
of EEG signal to achieve 97-99\% of accuracy. Zeynali and Seyedarabi [24] also reported that the O2 channel is the optimum channel placement for biometric EEG. They reach 95\% accuracy in a large dataset only with the O2 channel. Despite that, in [24], the EEG signal was collected with five mental activities. This study addresses some issues mentioned above, especially in data collection procedure and processing in single-channel EEG as a biometric modality. This study shows that it is possible to employ single-channel EEG as a biometric modality during resting EO conditions.

\section{RESEARCH METHOD}

\subsection{Data collection}

The raw EEG signals were collected using the Neurosky Mindwave Mobile headset. It uses a dry-type single-channel sensor placed at Fp1 according to the 10-20 placement system. The reference electrode was placed in the left ear. It was placed in the A1 position and a $512 \mathrm{~Hz}$ sampling rate was used. Nine participants participated in this research ( 8 males, 1 female, $M=20.66$ ). All participants had normal or corrected to normal vision, normal audition, and right-handed. All participants also never experienced any chronic disease. Considering the neurosky mindwave mobile headset uses a dry electrode, it doesn't require special preparation. It only took less than 10 seconds to prepare the equipment. Participants were asked to blink naturally during data acquisition. Each participant has performed five trial data collection procedures with 60 seconds each trial. 30s second resting time was given to participants was given between each trial. A total of 45 raw EEG signals were obtained in the data acquisition procedure.

\subsection{Methodology}

The proposed method is divided into three stages, preprocessing, feature extraction, and classification.

\subsubsection{Preprocessing}

The first step in the preprocessing stage is normalization. The raw signals were normalized into zero means with a minimum amplitude of -1 , and a maximum amplitude of 1 . In (1) and (2) were used to normalize the raw EEG signal.

$$
\begin{aligned}
& x_{\text {norm }}=\frac{x}{x_{\max }} \\
& x_{\max }= \begin{cases}|\max (x)| & \text { if }|\max (x)| \geq|\min (x)| \\
|\min (x)| & \text { if }|\max (x)|<|\min (x)|\end{cases}
\end{aligned}
$$

The second step was isolating the electrooculogram (EOG) signal from the EEG signal. The EOG signal is a signal generated by eye movement activity [23]. It contained in the EEG signal during the data collection procedure. It occurs because Fp1 was utilized as the main channel. The EOG signal generates more considerable electrical potential than the actual EEG signal. Therefore, it can be led into distorting information.

The most common method to isolate the EOG signal is independent component analysis (ICA). Conversely, ICA required multi-channel recorded EEG signals to produce an excellent result. As this research only used a single channel, it found it challenging to isolate the EOG signal using ICA. Therefore, this study employed the empirical model decomposition (EMD). EMD decomposes signal into several intrinsic mode functions (IMFs). EMD works great in isolation tasks according to [23]. Zahhad et al. [23] was found that the EEG signal concentrated in the first and second IMFs. However, [23] doesn't specify the maximum number of decomposed IMFs. There are no significant extrema at the $11^{\text {th }} \mathrm{IMF}$, according to author's observation based on collected signal. Therefore, the authors limit the total number of decomposed IMFs to 10 IMFs. Moreover, it found that the EEG signal concentrated only in the first IMFs. The remaining decomposed signals are identified as the EOG signal.

In the third step, the EEG signal is divided into non-overlapping $N$ segments. Suppiah and Vinod [15] used 5 seconds of the length of segments to divide the EEG signal. It showed a high correlation between the alpha band, power spectral density (PSD) feature, and accuracy in a relaxing state. In this study, 5 seconds of the segment were also employed. Therefore, 12 segments were extracted from each data. The alpha band $(8-12 \mathrm{~Hz})$ was extracted from each segment using the discrete wavelet transform (DWT). Daubechies type 4 (db4) was employed as a mother of wavelet. Furthermore, six levels of decomposition were performed to obtain the alpha band. As a result, $45 \times 12$ data points were collected in this step. 


\subsubsection{Features extraction}

Fast fourier transform (FFT) based on the Welch method was applied to estimate PSD [25]. PSD estimation is expressed in (3).

$$
\begin{aligned}
& \widehat{P}_{w e l c h}(f)=\frac{1}{L} \sum_{j=0}^{L-1} \hat{P}_{j}(f) \\
& \widehat{P}_{l}(f)=\frac{1}{K U}\left|w(n) x_{j}(n) e(-j 2 \pi f n)\right|^{2} \\
& w(n)=0.5\left(1-\cos \left(2 \pi \frac{n}{N}\right)\right), 0 \leq n \leq N
\end{aligned}
$$

$\widehat{P}_{J}(f)$ is the periodogram of each segment, where $j=1, \ldots, L-1$ are data segments, and $L$ is a number of segments. $\widehat{P}_{l}(f)$ estimation is given by (4). $K$ is the length of the segment. $U$ is the total average of $w(n) . w(n)$ is the window function as expressed in (5). Hanning window was applied in this study. The number of overlapping windows used in this research is $50 \%$. A total of $45 \times 12 \times 84$ data points were extracted in the feature extraction stage.

Moreover, the dimensional reduction procedure was applied. Principal component analysis (PCA) and linear discriminant analysis (LDA) are known as adequate dimensional reduction methods. However, PCA works well with unlabeled data. Therefore, LDA was employed as a dimensional reduction method. As a sparse solver, singular value decomposition (SVD) and LDA maximize the dimensional reduction method. The dimensional reduction procedure reduced the number of features vector to $45 \times 12 \times 8$.

\subsubsection{Classification}

LDA using the least-square solver and support vector machine (SVM) using linear kernel were applied in the classification stage. Cross-validation was employed to split data into training and testing sets. The number of $k=10$ was employed. Furthermore, the classification stage was performed in three main conditions. The first condition is classification by using the combination of all segments. The second condition is the classification of each segment. The third condition is a combination of segments. In the combination of segments, all segments were divided into three sections with an equal number of segments, the first section, the middle section, and the last section. Each section consists of 4 segments. The combination of segments from the different sections is also employed in the classification stage.

Table 1 shows the combination of segments that were used in this study. Group G1, G2, and G3 are the groups from the segments in the first, middle, and last section, respectively. Group G4 combines the first segments from each section. Group G5, G6, and G7 follow the G4 segment combination rule, although they use the second, the third, and the fourth segments, respectively. The intention of employed segments combination is to find a specific segment that may contain primary information. Thus, it can reduce the computational cost compared to the use of all segments.

Table 1. Groups of segment combination

\begin{tabular}{cc}
\hline Group Name & Segment Combination \\
\hline G1 & $1,2,3,4$ \\
G2 & $4,5,6,7$ \\
G3 & $9,10,11,12$ \\
G4 & $1,5,9$ \\
G5 & $2,6,10$ \\
G6 & $3,7,11$ \\
G7 & $4,8,12$ \\
\hline
\end{tabular}

\section{RESULTS AND DISCUSSION}

Figure 1 shows the classification result from LDA and SVM using all segments. 78\% and 70\% accuracy achieved using LDA and SVM, respectively. Based on those accuracies, it showed unsatisfactory results for the biometric system. Hence, the analysis based on each segment was performed. Figure 2 shows the classification result that employed each segment.

The LDA showed better results compared to the SVM. It also found that the average accuracy of each segment showed better accuracy compared to the accuracy of all segments. The LDA obtained $82.58 \%$ accuracy, although the SVM obtained $76.67 \%$ accuracy, as shown in Figure 3. The best accuracy was found at segment number 3 and number 11 with $86 \%$ accuracy using LDA, as shown in Figure 2. Nonetheless, in 
the SVM classifier, the best accuracy also leans on segment number 3. The result indicated that the main information might be concentrated in segment number 3 .

Moreover, the performance of each group of segment combinations was inspected. The classification result is shown in Figure 4. The best accuracy was obtained by group G1 with $82 \%$ accuracy. Based on Table 1 , G1 consists of the $1^{\text {st }}, 2^{\text {nd }}, 3^{\text {rd }}$, and $4^{\text {th }}$ segments. The result of G1 might be influenced by segment number 3. However, the finding of segment number 3 may increase the accuracy of G1 was not following the result of G6. The G6 only got $68 \%$ by using LDA and $59 \%$ by using SVM. Looking further, G6 consists of segments 3, 7, and 11. As already shown in Figure 2, segments 3 and 11 resulted in the highest accuracy using LDA. They also resulted in the first and second-best accuracy using SVM. Besides, segment numbers 7 and 10 were the least accurate. It also found that segment number 10 was the second least accuracy with $79 \%$ accuracy by using LDA.

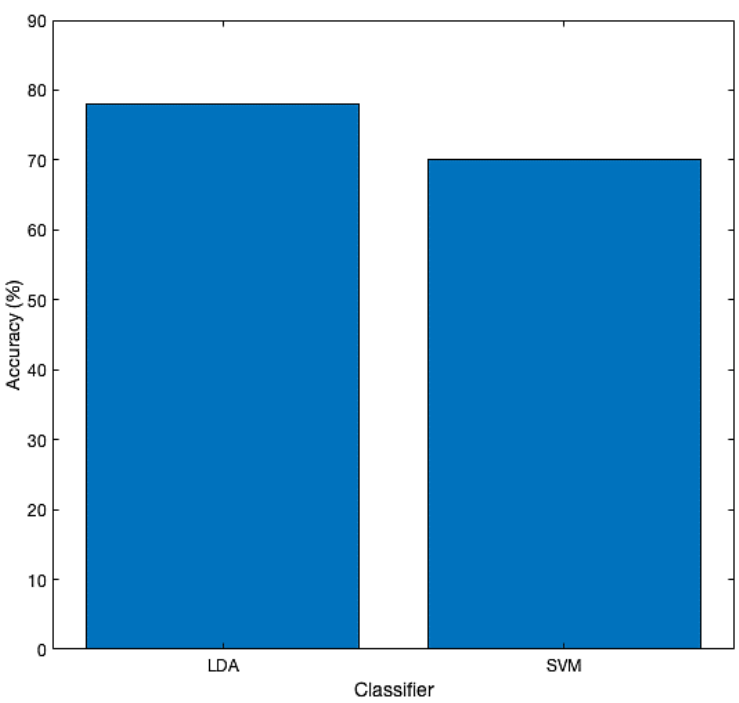

Figure 1. Classification result from all segments

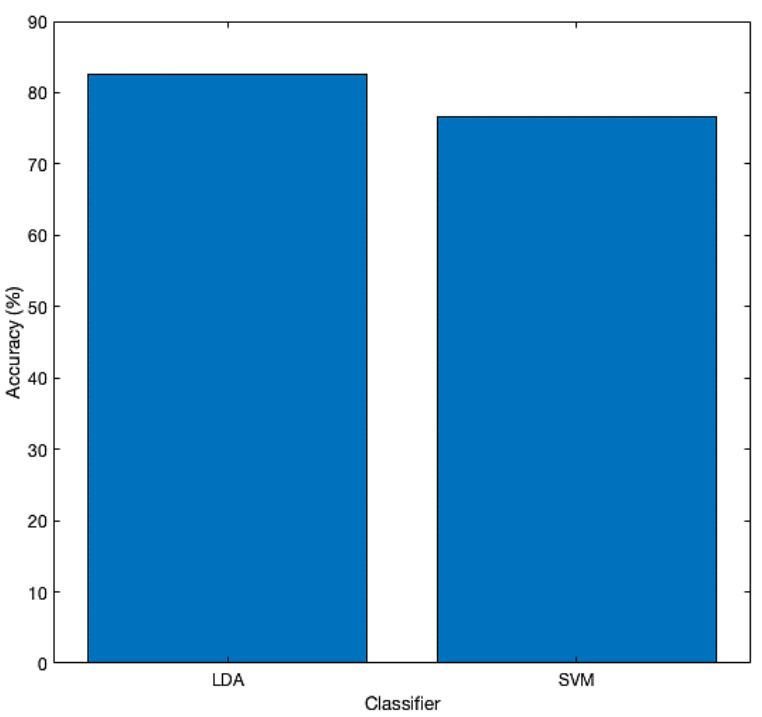

Figure 3. Average accuracy from all segments

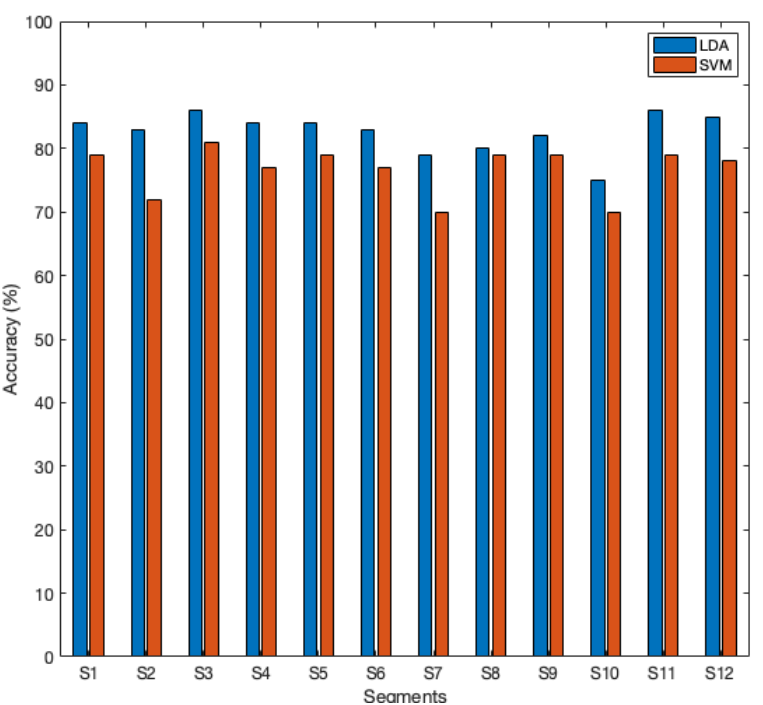

Figure 2. Classification result for each segment

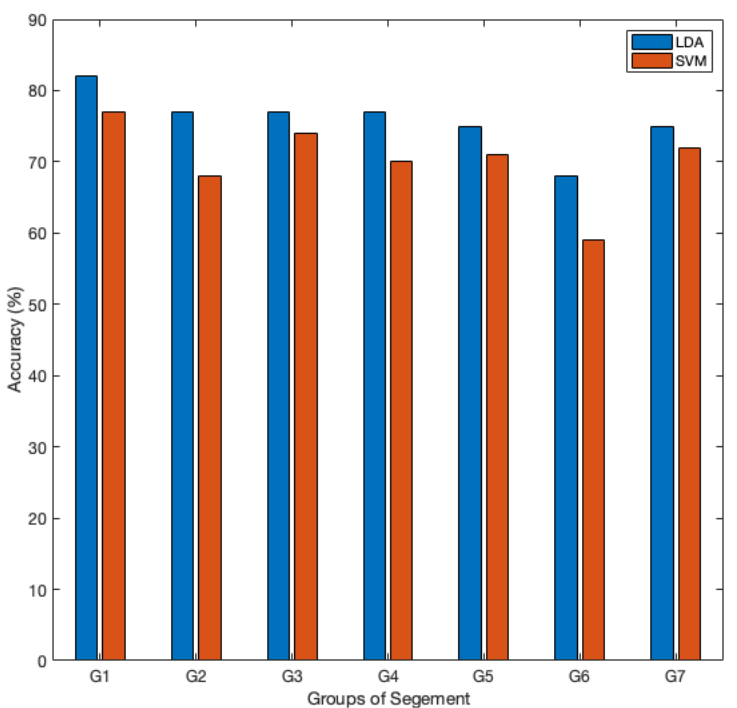

Figure 4. Classification result from each group

Furthermore, the new groups of segment combinations were analyzed. Table 2 shows the new combination of segments. The new groups were created based on the best segment accuracy from each section. Segment numbers 3, 5, and 11 were the best segments from each section, respectively, in the LDA,

\footnotetext{
Identification of optimum segment in single channel EEG biometric system (Muhammad Afif Hendrawan)
} 
although the SVM made more combinations as there are segments that had the same accuracy in the same section. The new groups allowed a test of the hypothesis that the combination of the best segments might increase the accuracy. The highest accuracy was achieved by G8 and G11 using LDA, as shown in Figure 5. Both G8 and G11 consist of segments 3 and 11. However, the result of G8 and G11 didn't exceed the accuracy of segments 3 and 11. Therefore, the features that lean on segments 3 and 11 may be use in the EEG biometric system due to the highest accuracy.

The study reveals the possibility of single-channel EEG as a modality in the biometric system, especially in the resting eye open condition. The proposed method was more suitable in real conditions. This study also presents a segmentation analysis in EEG processing as a biometric modality. In this study, segment number 3 showed the highest accuracy. It demonstrates that the proposed EEG biometric requires only 15 seconds to distinguish one person from others with reasonable accuracy. Additionally, the small amount of data used in the proposed method may cause lower computational costs.

Table 2. New groups of segment combination

\begin{tabular}{cc}
\hline Group Name & Segment Combination \\
\hline G8 & $3,5,11$ \\
G9 & $3,5,9$ \\
G10 & $3,8,9$ \\
G11 & $3,8,11$ \\
\hline
\end{tabular}

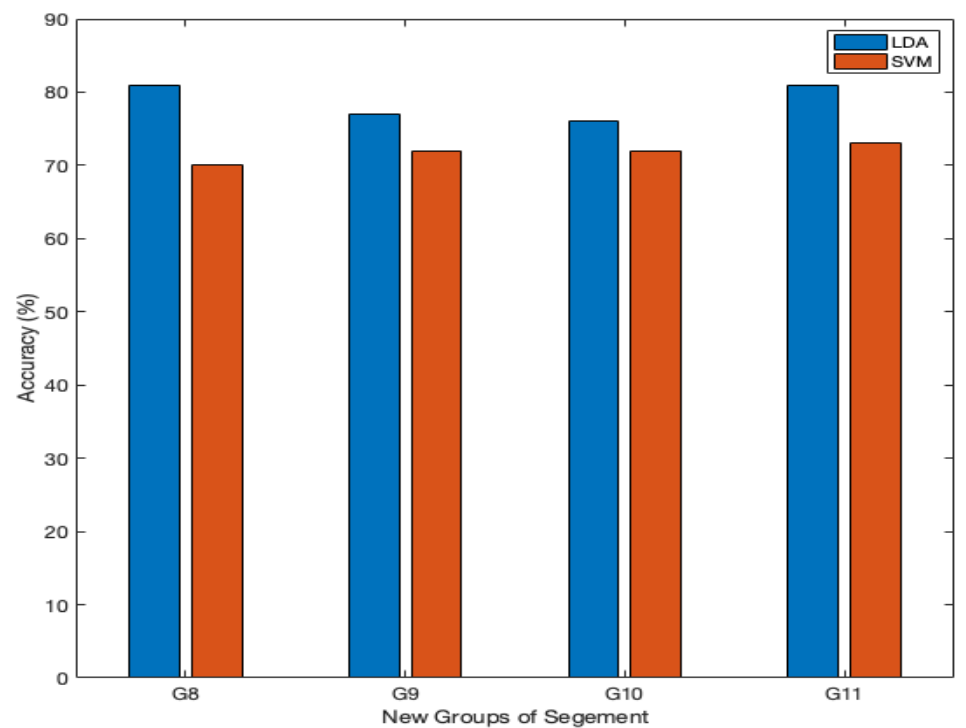

Figure 5. Classification result of the new groups of segments

\section{CONCLUSION}

This paper studied the possibility of single-channel EEG as a biometric modality. The proposed method combined the EOG rejection and segmentation paradigm to provide a fair precision and reliable biometric system. The EOG rejection method allowed the data collection procedure to be performed while the subject was resting with eye open condition. It was closer to the real condition where the subject wasn't required to perform any other action, such as motor imaginary, visual imaginary, or even prevent blinking activity. The segmentation paradigm shows that the main features might lean on a specific segment. Therefore, we are able to use a specific segment to provide a high-quality biometric system. Accuracy of 86\% was obtained from 15 seconds of recorded EEG. It took longer than a conventional biometric system, such as a fingerprint system. However, it shows that EEG as a modality of a biometric system is promising to provide a high-security system with a low computational cost. In the future, we will investigate the same method on a large amount of data. The overlapping segment will be investigated. Moreover, EEG signal consistency is a major issue in the study of biometrics. We are also will to investigate this issue regarding the possibility of the EEG signal that may change as a human gets older. 


\section{REFERENCES}

[1] I. Jayarathne, M. Cohen, and S. Amarakeerthi, "Survey of EEG-based biometric authentication," in Proceedings 2017 IEEE 8th International Conference on Awareness Science and Technology, iCAST 2017, vol. 2018-Janua, no. iCAST, 2017, pp. 324-329, doi: 10.1109/ICAwST.2017.8256471.

[2] A. S. Raju and V. Udayashankara, "Biometric person authentication: A review," Proceedings of 2014 International Conference on Contemporary Computing and Informatics, IC3I 2014, no. d, 2014, pp. 575-580.

[3] K. Dharavath, F. A. Talukdar, and R. H. Laskar, "Study on biometric authentication systems, challenges and future trends: A review," 2013 IEEE International Conference on Computational Intelligence and Computing Research, IEEE ICCIC 2013, 2013, doi:10.1109/ICCIC.2013.6724278.

[4] D. Maltoni, D. Maio, A. K. Jain, and S. Prabhakar, "Handbook of Fingerprint Recognition," 2nd ed. London: Springer London, 2009.

[5] D. A. Reynolds et al., "The 2004 MIT Lincoln Laboratory speaker recognition system," ICASSP, IEEE International Conference on Acoustics, Speech and Signal Processing - Proceedings, vol. I, 2005, pp. 177-180, doi: 10.1109/ICASSP.2005.1415079.

[6] J. Daugman, "How Iris Recognition Works," The Essential Guide to Image Processing, vol. 14, no. 1, pp. 715-739, 2009.

[7] T. Matsumoto, H. Matsumoto, K. Yamada, and S. Hoshino, "Impact of artificial "gummy" fingers on fingerprint systems," Proceedings of SPIE - The International Society for Optical Engineering, vol. 4677, no. 1, pp. 275-289, 2002, doi: $10.1117 / 12.462719$.

[8] K. A. Nixon, V. Aimale, and R. K. Rowe, "Spoof Detection Schemes," in Handbook of Biometrics, 2007.

[9] R. B. Navarro, L. B. Vázquez, and E. L. Guillén, "EOG-based wheelchair control," in Smart Wheelchairs and Brain-computer Interfaces: Mobile Assistive Technologies, 2018.

[10] C. Rahmad, R. Ariyanto, and D. R. Yunianto, "Brain signal classification using Genetic Algorithm for right-left motion pattern," International Journal of Advanced Computer Science and Applications, vol. 9, no. 11, 2018, doi: 10.14569/IJACSA.2018.091134.

[11] S. Y. Gordleeva et al., "Real-Time EEG-EMG human-machine interface-based control system for a lower-limb exoskeleton," IEEE Access, vol. 8, pp. 84070-84081, 2020, doi: 10.1109/ACCESS.2020.2991812.

[12] I. Odinaka, P. H. Lai, A. D. Kaplan, J. A. O’Sullivan, E. J. Sirevaag, and J. W. Rohrbaugh, "ECG biometric recognition: A comparative analysis," IEEE Transactions on Information Forensics and Security, vol. 7, no. 6, pp. 1812-1824, 2012, doi: 10.1109/TIFS.2012.2215324.

[13] S. Marcel and J. del R. Millan, "Person authentication using brainwaves (EEG) and maximum a posteriori model adaptation," IEEE Transactions on Pattern Analysis and Machine Intelligence, vol. 29, no. 4, pp. 743-748, 2007, doi: 10.1109/TPAMI.2007.1012.

[14] M. Del Pozo-Banos, J. B. Alonso, J. R. Ticay-Rivas, and C. M. Travieso, "Electroencephalogram subject identification: A review," Expert Systems with Applications, vol. 41, no. 15, pp. 6537-6554, 2014, doi: 10.1016/j.eswa.2014.05.013.

[15] R. Suppiah and A. P. Vinod, "Biometric identification using single channel EEG during relaxed resting state," IET Biometrics, vol. 7, no. 4, pp. 342-348, 2018.

[16] R. B. Paranjape, J. Mahovsky, L. Benedicenti, and Z. Koles, "The electroencephalogram as a biometric," CANADIAN Conference on Electrical and Computer Engineering, vol. 2, pp. 1363-1366, 2001, doi: 10.1109/CCECE.2001.933649.

[17] R. Palaniappan and D. P. Mandic, "Biometrics from brain electrical activity: A machine learning approach," IEEE Transactions on Pattern Analysis and Machine Intelligence, vol. 29, no. 4, pp. 738-742, 2007, doi: 10.1109/TPAMI.2007.1013.

[18] G. Schalk, D. J. Mcfarland, T. Hinterberger, N. Birbaumer, and J. R. Wolpaw, "BCI2000 : A General-Purpose Brain-Computer Interface (BCI) System," IEEE Transactions on Biomedical Engineering, vol. 51, no. 6, pp. 1034-1043, 2004, doi: 10.1109/TBME.2004.827072.

[19] D. La Rocca et al., "Human brain distinctiveness based on EEG spectral coherence connectivity," IEEE Transactions on Biomedical Engineering, vol. 61, no. 9, pp. 2406-2412, 2014, doi: 10.1109/TBME.2014.2317881.

[20] M. Fraschini, A. Hillebrand, M. Demuru, L. Didaci, and G. L. Marcialis, "An EEG-based biometric system using eigenvector centrality in resting state brain networks," IEEE Signal Processing Letters, vol. 22, no. 6, pp. 666-670, 2015, doi: 10.1109/LSP.2014.2367091.

[21] K. P. Thomas and A. P. Vinod, "Biometric identification of persons using sample entropy features of EEG during rest state," 2016 IEEE International Conference on Systems, Man, and Cybernetics, SMC 2016 - Conference Proceedings, 2017, pp. 3487-3492, doi: 10.1109/SMC.2016.7844773.

[22] J. H. Kang, Y. C. Jo, and S. P. Kim, "Electroencephalographic feature evaluation for improving personal authentication performance," Neurocomputing, vol. 287, pp. 93-101, 2018, doi: 10.1016/j.neucom.2018.01.074.

[23] M. Abo-Zahhad, S. M. Ahmed, and S. N. Abbas, "A novel biometric approach for human identification and verification using eye blinking signal," IEEE Signal Processing Letters, vol. 22, no. 7, pp. 876-880, 2015, doi: 10.1109/LSP.2014.2374338.

[24] M. Zeynali and H. Seyedarabi, "EEG-based single-channel authentication systems with optimum electrode placement for different mental activities," Biomedical Journal, vol. 42, no. 4, pp. 261-267, 2019, doi: 10.1016/j.bj.2019.03.005.

[25] P. D. Welch, "The Use of Fast Fourier Transform for the Estimation of Power Spectra: A Method Based on Time Averaging Over Short, Modified Periodograms," IEEE Transactions on Audio and Electroacoustics, vol. 15, no. 2, pp. 70-73, 1967, doi: 10.1109/TAU.1967.1161901. 


\section{BIOGRAPHIES OF AUTHORS}

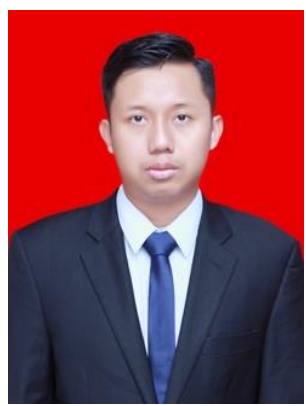

Muhammad Afif Hendrawan received bachelor degree from Department of Information Systems, Institut Teknologi Sepulurh Nopember in 2015 and master degree from Department of Electrical Engineering, Institut Teknologi Sepuluh Nopember in 2017. His currenly working as assistant professor at Politeknik Negeri Malang. His research interest is in the area of artificial intelligence, edge computing, signal processing, and brain-computer interface.

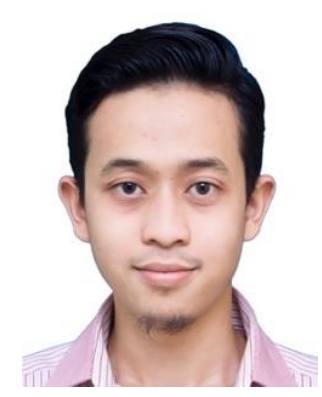

Pramana Yoga Saputra received bachelor degree from Department of Informatics Engineering, Institut Teknologi Sepuluh Nopember in 2010 and master degree from Department of Technology Management, Instititut Teknologi Sepuluh Nopember in 2014. His research interest is human-computer interaction, artificial intelligence, and natural language processing.

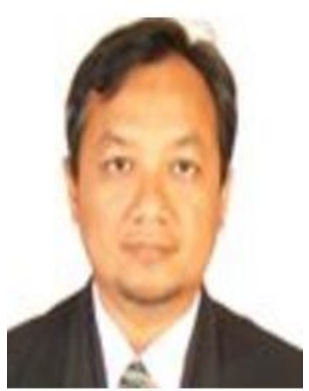

Cahya Rahmad received BS degree from Brawijaya Univerisity in 1998 and MS degree from Department of Informatics Engineering, Institut Teknologi Sepuluh Nopember in 2005. He received a doctoral degree from Saga University, Japan, in 2013. He is a lecturer in Politeknik Negeri Malang. His research interest are image processing, data mining, pattern recognition, artificial intelligence, and brain-computer interface. 